Research Paper

\title{
Bone Regeneration using Silk Hydroxyapatite Hybrid Composite in a Rat Alveolar Defect Model
}

\author{
Kyung S. Koh, Jong Woo Choi, Eun Jeong Park, Tae Suk Oh ${ }^{\bowtie}$ \\ Department of Plastic Surgery, Asan Medical Center and University of Ulsan College of Medicine \\ $\square$ Corresponding author: Tae Suk Oh, M.D., Ph.D., Clinical Assistant Professor, Department of Plastic Surgery, Asan Medical Center and University of Ulsan \\ College of Medicine, 388-1 PungNap-2Dong, SongPa-Gu, 138-736, Seoul, Korea tasuko@amc.seoul.kr, phone: 82-2-3010-3600/fax: 82-2-476-7471 \\ (c) Ivyspring International Publisher. This is an open access article distributed under the terms of the Creative Commons Attribution (CC BY-NC) license \\ (https://creativecommons.org/licenses/by-nc/4.0/). See http://ivyspring.com/terms for full terms and conditions.
}

Received: 2017.07.05; Accepted: 2017.10.11; Published: 2018.01.01

\begin{abstract}
Background: To overcome the limited source of autogenous bone in bone grafting, many efforts have been made to find bone substitutes. The use of hybrid composites of silk and hydroxyapatite to simulate natural bone tissue can overcome the softness and brittleness of the individual components.

Methods: Critical-sized, $7 \times 4 \times 1.5 \mathrm{~mm}$ alveolar defects were created surgically in 36 Sprague-Dawley rats. Three treatment groups were tested: an empty defect group (group I), a silk fibrin scaffold group (group II), and a hydroxyapatite-conjugated silk fibrin scaffold group (group III). New bone formation was assessed using computed tomography and histology at 4, 8, and 12 weeks, and semi-quantitative western blot analysis was done to confirm bone protein formation at 12 weeks. Statistical analysis of new bone formation was done using the Kruskal-Wallis test.

Results: Radiomorphometric volume analysis revealed that new bone formation was $64.5 \%$ in group I, $77.4 \%$ in group II, and $84.8 \%$ in group III $(p=0.027)$ at 12 weeks. Histologically, the osteoid tissues were surrounded by osteoblasts not only at the border of the bone defect but in the center of the scaffold implanted area in group III from week 8 on. Semi-quantitative western blotting revealed that osteocalcin expression in group III was 1.8 times higher than group II and 2.6 times higher than group I.

Conclusions: New bone formation was higher in hybrid scaffolds. Both osteoconduction at the defect margin and osteoinduction at the center of the defect were confirmed. There were no detected complications related to foreign body implantation.
\end{abstract}

Key words: alveolar bone defect, bone regeneration, silk scaffold, hydroxyapatite.

\section{Introduction}

The grafted bone survival rate for an alveolar bone defect is $41 \%$ to $73 \% .^{1-3}$ Cancellous bone of the iliac area is mainly used as donor material. Possible complications include wounding at the donor site, postoperative hematoma, infection, and gait disturbances. Moreover, when the alveolar bone defect is large, several bone grafts are necessary. The risk of complications in the donor area increases accordingly with increased need to use cancellous bone from both sides of iliac area. ${ }^{1-3}$ Due to these risks, it is necessary to find a replacement for autogenous bone. Research and development of many substances are currently underway.
The regeneration of insufficient tissue requires three tissue engineering elements; cells, a scaffold, and signaling elements such as growth factors. In our current study, an organic/inorganic hybrid compound of silk and hydroxyapatite was used as the scaffold. ${ }^{4-6}$ Hydroxyapatite is a representative substance used in various fields for scaffolding bone defects due to its capacity for osteoinduction. Silk, created from Bombyx mori, has been used as a suture material for a long time due to its superior biocompatibility, proven through testing of its biological safety and biodegradability.7-13 However, silk alone lacks the mechanical strength needed to 
replace bone tissue, and hydroxyapatite may break upon impact when used by itself, despite its hardness. In order to overcome the disadvantages of the organic and inorganic materials of silk and hydroxyapatite, a study on the use of a hybrid composite of these two substances to replace bone tissue was previously conducted. ${ }^{14}$

Kaplan et al. conducted a study on the physical properties of a hybrid scaffold composed of silk and hydroxyapatite. ${ }^{15}$ They suggested that hydroxyapatite is a substance with outstanding biocompatibility and bioactivity and it is substituted with growing bone through the osteoinduction process after grafting. Bone regeneration using a silk scaffold combined with hydroxapatite occurs via two processes, osteoconduction from the surrounding bone in the defect area and nucleation with the combined hydroxyapatite as its seed. This is significant because bone regeneration using the hybrid composite is faster than regeneration by the surrounding bone, resulting in consistent ossification in all areas, including the center of the bone defect. ${ }^{15-19}$

Direct insertion of hydroxyapatite in liquid form or a direct graft after dipping into a collagen scaffold results in serious disadvantages, including unexpected whole-body effects and side effects due to inflow to the blood and uncontrolled biochemical activation. To overcome these shortcomings, a hybrid scaffold of silk and hydroxyapatite was grafted to the alveolar bone of Sprague-Dawley rats with critical-size bone defects, allowing for continuous biochemical activation and preventing inflow into the blood stream.

\section{Materials and Methods}

\section{Alveolar Bone Defect Formation in Sprague-Dawley Rats}

Thirty-six male Sprague-Dawley rats of 9 to 10 weeks of age and weighing 240-250g were used as experimental animals in this study. Experiments were conducted with the permission of the Animal Testing Ethics Committee of the Clinical Study Center at the Asan Medical Center, Seoul, South Korea. The animals were managed based on the regulations specified by this Committee. Three groups were classified based on the materials used for grafting the generated alveolar bone defect. The animals in group I were sutured without a scaffold bone graft $(n=12)$. The animals in group II were sutured after a silk scaffold graft $(n=12)$. The animals in group III were grafted with a hybrid scaffold of silk and hydroxyapatite $(n=12)$.

To create bone defects, 9-week-old Sprague-Dawley rats were placed in the supine position and administered anesthesia with an intraperitoneal injection of Zoletil ${ }^{\circledR}$. A $7 \times 4 \times 1.5 \mathrm{~mm}$ bone defect was created by making a $1 \mathrm{~cm}$ incision toward the longitudinal direction in the mucous membrane between the hard palate of the right upper jaw and the alveolar bone and exposing the alveolar bone by dissecting its periosteum after exposure (Fig. 1).

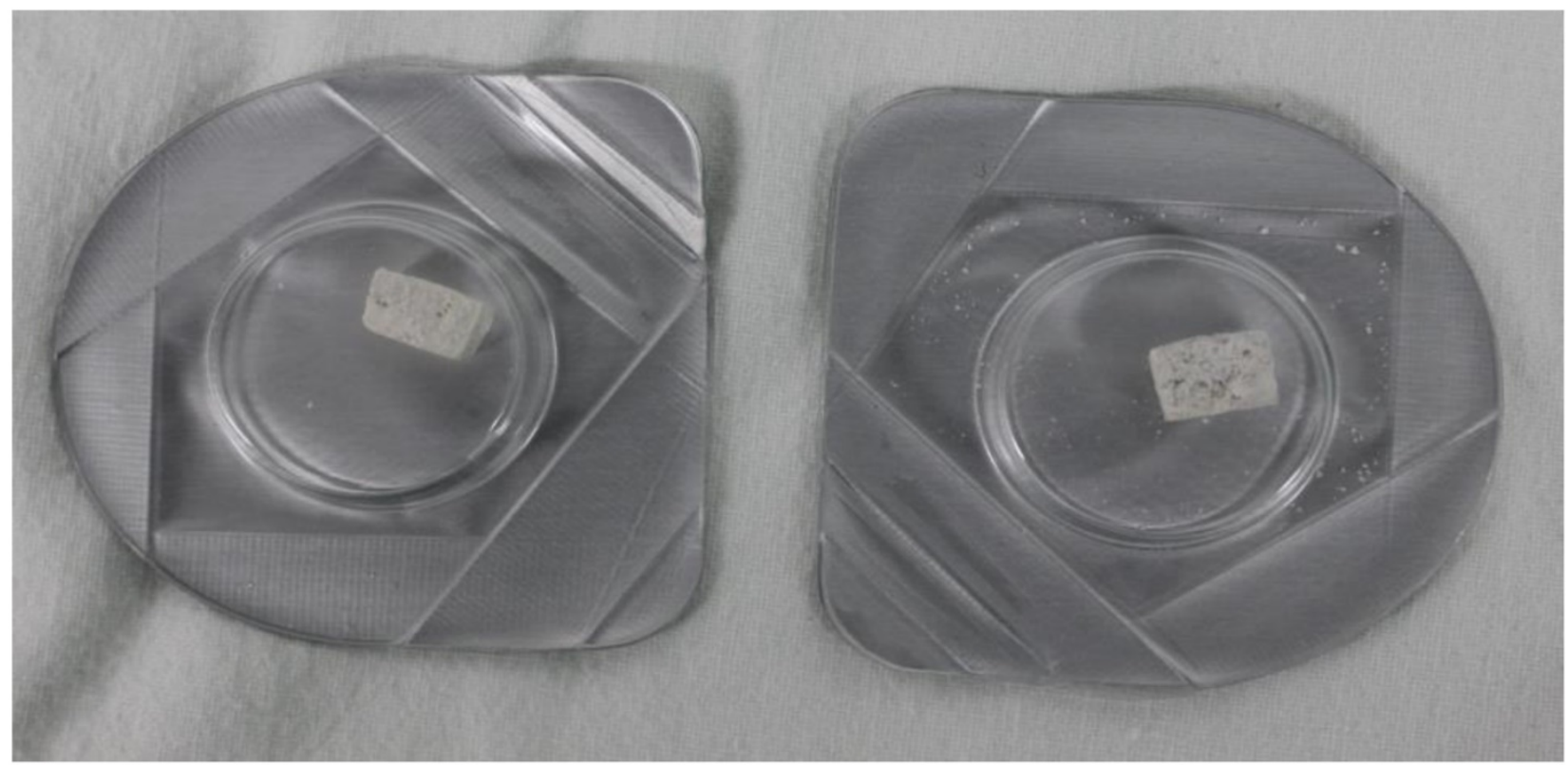

Figure 1. Hybrid scaffold of silk and hydroxyapatite 


\section{Manufacturing the Silk Scaffold with Hydroxyapatite}

Silk consists of a 7:3 ratio of fibroin and sericin. Its physical properties differ with the amino acid composition and fibroin/sericin content. Silk scaffolds are manufactured by removing the sericin to isolate and retain only the fibroin and acquire its biocompatibility, oxygen and moisture penetrability, cytotropism, and biodegradability. Specifically in our present study, the silk fibroin solution was manufactured by removing sericin using a $>90^{\circ} \mathrm{C}$ $\mathrm{Na}_{2} \mathrm{CO}_{3}$ solution, refining the silk, and creating an $8-20 \%$ silk solution with the use of solvent $(\mathrm{LiBr}$ solution or $\mathrm{CaCl}_{2}$ /Ethanol/water mixture). A dialysis process was used to remove the salt component of the solvent. The silk scaffold was manufactured using such a solution, adding salt, leaving it at room temperature, creating a crystal, dipping the crystal into water to remove the salt, and drying it upon the completion of salt removal (BioAlpha, Inc., Seoul, Korea). The manufactured silk scaffold was mixed together with granular hydroxyapatite at a 10:1 ratio and sterilized by irradiating with gamma rays after freeze-drying for three days (BioAlpha, Inc., Korea) (Fig. 2, 3).

\section{Silk Scaffold Grafting}

For the graft, the scaffold was dipped into saline solution $(0.9 \% \mathrm{NaCl})$ for 30 minutes to allow manipulation into the shape of the bone defect area. After cutting the pre-treated scaffold to the same size as the bone defect, it was grafted to the critical-size $7 x$
$4 \times 1.5 \mathrm{~mm}$ bone defect area that was previously created using a power drill. The mucous membrane was then sutured using 4-0 black silk (Fig. 4).

\section{Assessment}

Assessments were conducted 4, 8, and 12 weeks after grafting the silk scaffold. Gross inspection, tissue analysis, CT of the bone defect, and other analyses were conducted to view new bone regeneration. Western blot analysis was conducted at week 12 to compare the degree of bone generation.

New Bone Yield Rate $(\%)=100 \times$ (Volume of initial bone defect - Measured volume of bone defect)/ Volume of initial bone defect (\%)

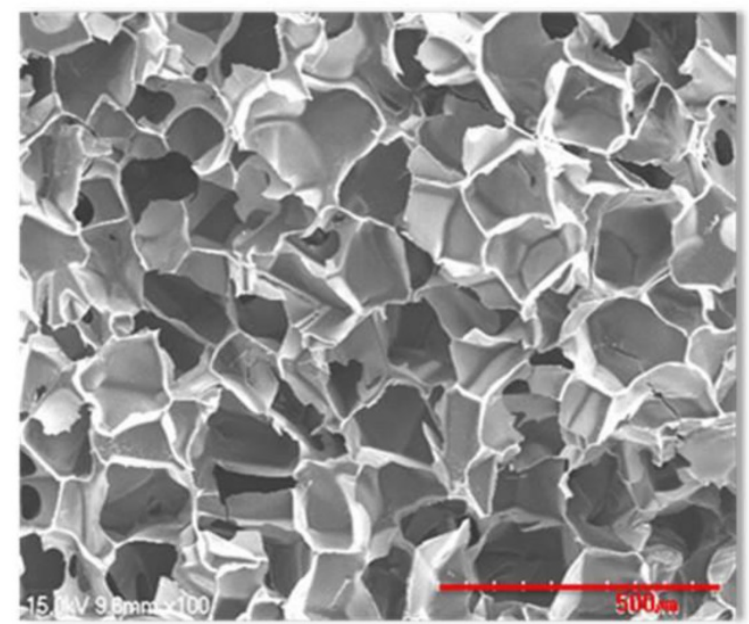

Figure 2. Scanning microscopic images of hydroxyapatite silk fibroin composites

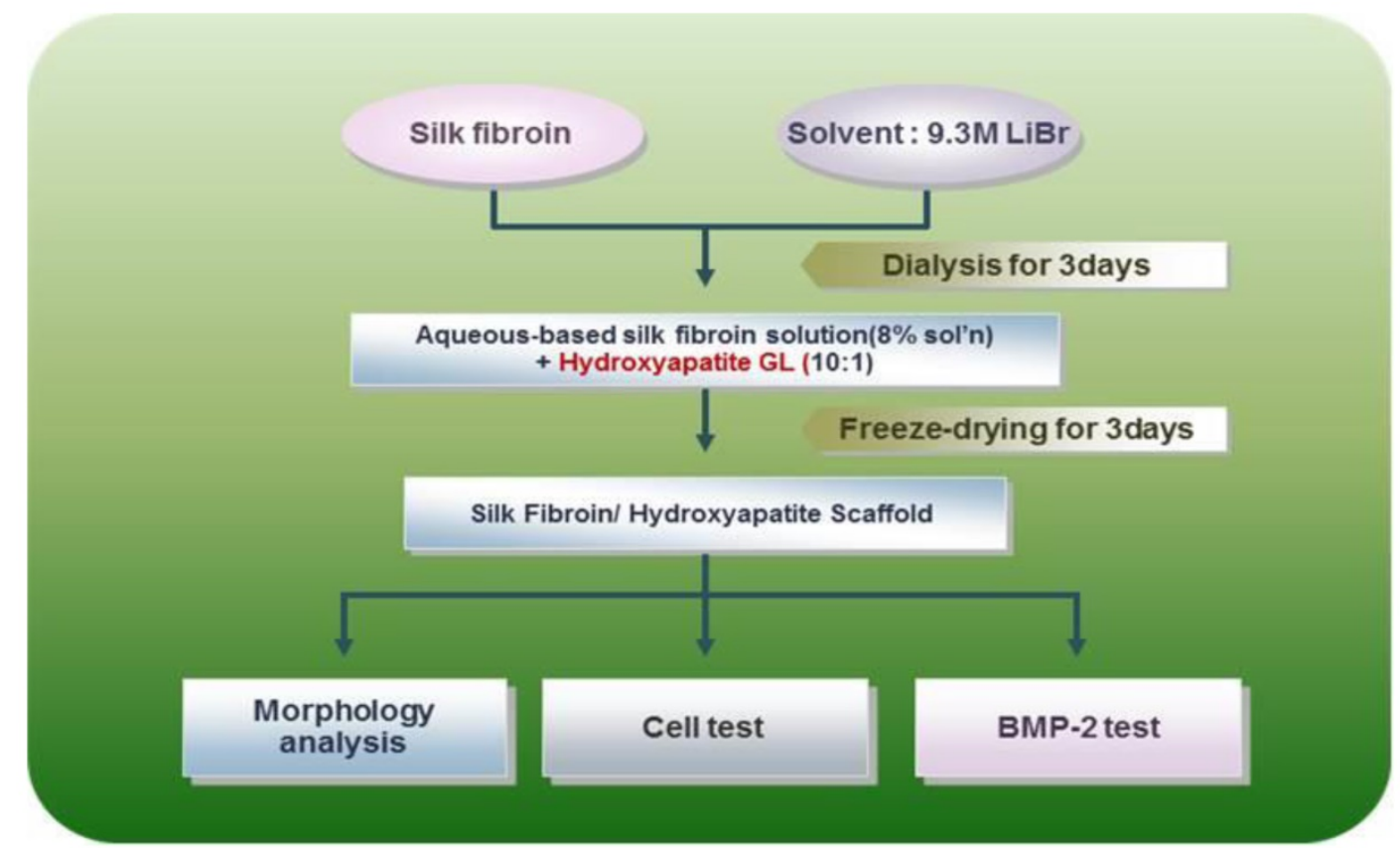

Figure 3. The main producing process of hybrid scaffold of silk and hydroxyapatite. The manufactured silk scaffold was mixed together with granular hydroxyapatite at a 10:1 ratio and freeze dried for 3 days. 


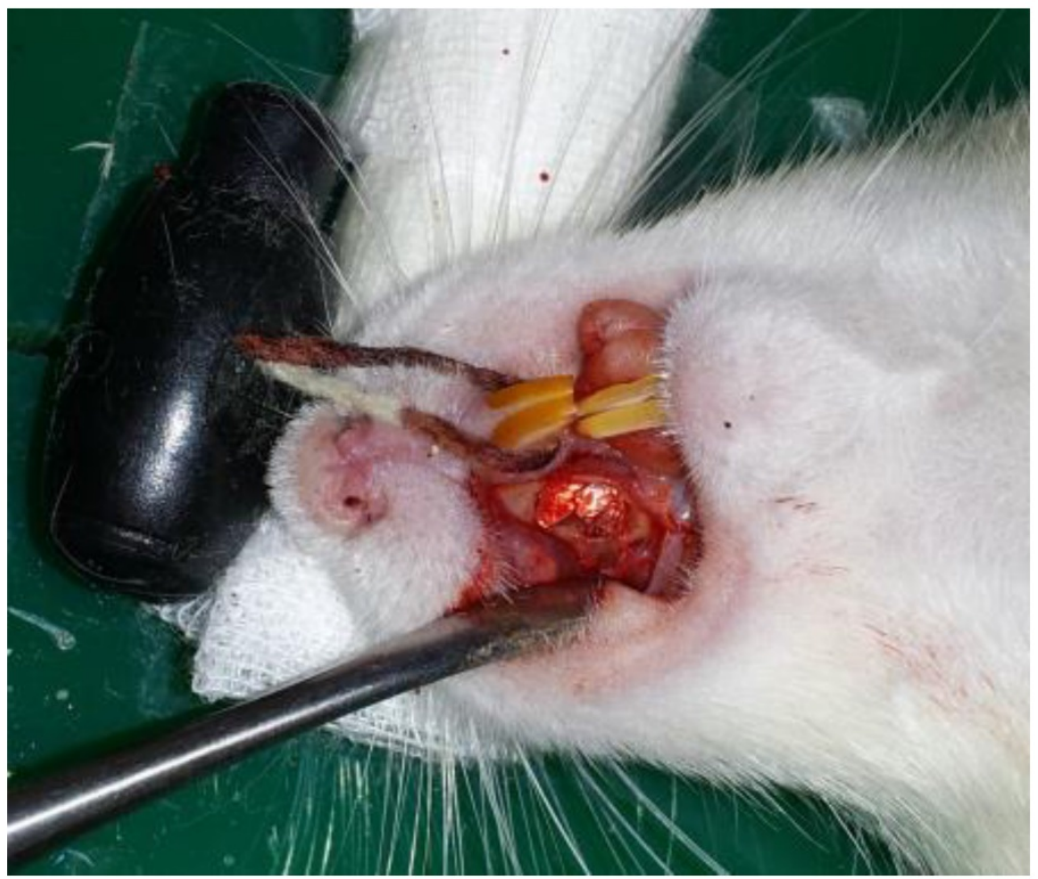

Figure 4. Hybrid scaffold grafted to the bone defect in a rat model

\section{Statistical Analysis}

The bone defect volumes quantified through CT are presented as the mean \pm standard deviation. The Kruskal-Wallis test was used for overall comparison of the 3 groups, and the Mann-Whitney test using the Bonferroni correction was used to compare results from 2 groups. A p-value of less than 0.05 was considered to be statistically significant, and the significant $\alpha$ value was set as 0.0167 for the Bonferroni correction. Analysis of all data was conducted using SPSS version 15.0 (SPSS, Inc., Chicago, IL).

\section{Results}

\section{Visual Inspection}

The grafted part of the upper jaw alveolar bone area was re-dissected at weeks 4,8 , and 12 after the scaffold grafting and inspected for changes. By eye, no ossification was observed at the center of the graft in any group at week 4 . The graft was covered with granulation tissue, and a hematoma in the bone defect area was observed in one group I case. In group III, hydroxyapatite still remained in its granular shape, confirming that no progress in ossification had occurred. There was an increase in ossification surrounding the bone defect at week 8 . In particular, considerable ossification was seen in group III. Both ossification through osteoinduction in the area surrounding the bone defect and ossification at the center of the bone defect were confirmed at week 12 .
In group III, the ossification could be confirmed by eye in most of the bone defect areas.

\section{Tissue Analysis}

After decalcification, the tissues were observed using an optical microscope after hematoxylin and eosin (H\&E) staining. An increase in granulation tissue with collagen fiber was confirmed in the area surrounding the scaffold in most groups at week 4 . Bone regeneration was only found in the area surrounding the bone defect, and osteoblasts were not observed at the center of the bone defect (Fig. 5). At week 8, primary bone tissue surrounded by osteoblasts was confirmed at the center of the bone defect in group III. At week 12, a large quantity of mature bone tissue was observed in both the area surrounding the bone defect and the center of the defect.

\section{Analysis of Quantified New Bone Volume Using CT}

Bone regeneration was observed only in the area surrounding the bone defect in most of the groups at week 4, and it was not shown at the center of the bone defect. More progress was confirmed in bone regeneration by osteoconduction from the boundaries of the bone defect at week 8 . The newly formed bone tissue was observed in the center of the bone defect through a cross-section of the CT, especially in group III. The increase in bone tissue was observed both in the area surrounding the bone defect and in the center of the defect at week 12 . 


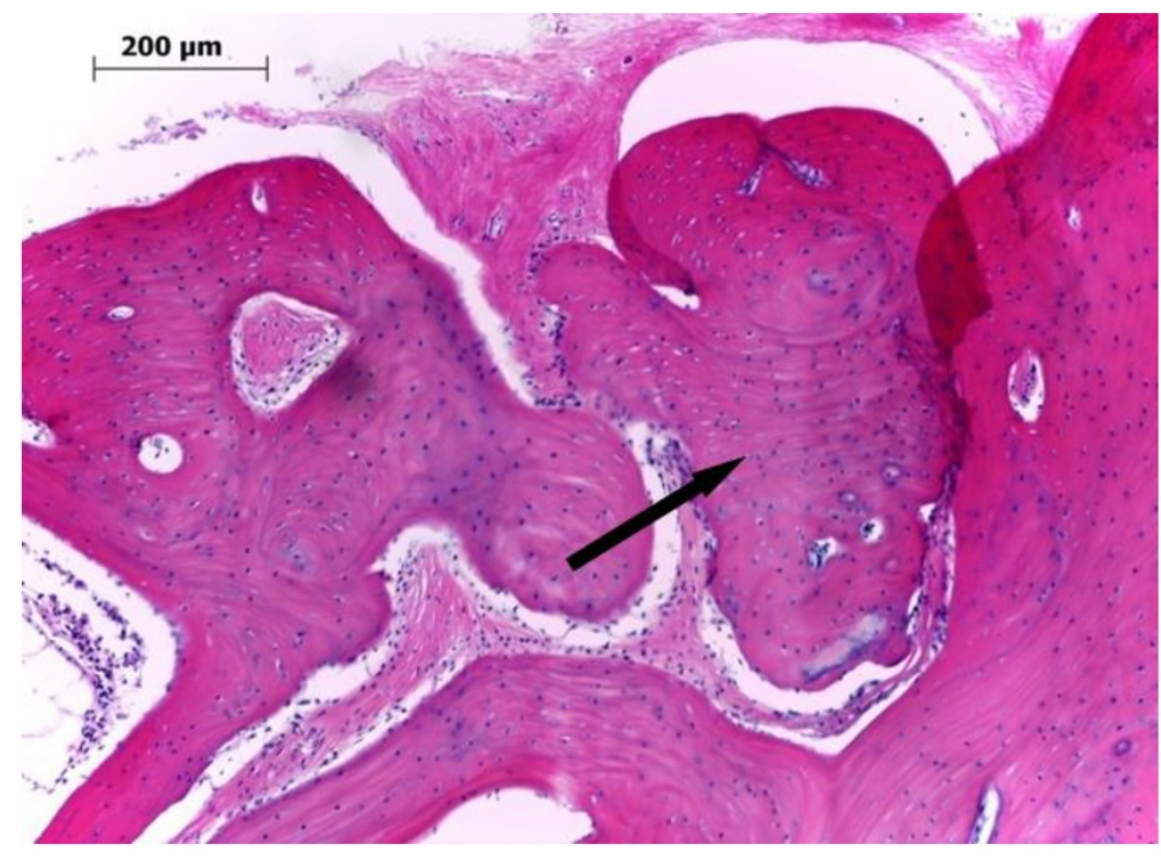

Figure 5. Microscopic findings for the bone defect in group III at 12 weeks after the graft of the hybrid scaffold (H\&E $\times 100)$. The extracellular environment including fibrous collagen, was mostly changed into lamellar bone, and there was an increase in the thickness of the mature bone (arrow).

The volume of the bone defect and the regeneration yield rate of the new bone were calculated with the above-mentioned method using a three-dimensional reconstructed bone defect image (Figs. 7 and 8). The results showed that $49.1 \%, 56.2 \%$, and $63.8 \%$ of new bone regeneration was achieved at week 4 in groups I to III, respectively $(p=0.058)$. At week 8 , the bone regeneration values were $56.3 \%$, $59.7 \%$, and $74.2 \%$ in groups I to III, respectively $(p=0.061)$. At week 12, the bone regeneration values were $64.5 \%, 77.4 \%$, and $84.8 \%$ for groups I to III, respectively $(p=0.027)$. From the cross-section image analysis by $\mathrm{CT}$ at week 12 , both bone regeneration from the boundary of the bone defect and radiolucency of the surrounding area at the center of the bone were clearly observed in group III. A maximum value of 359 for the Hounsfield number was found, which was relatively low compared to the values of 600 to 800 found in the surrounding bone.

\section{Quantification of Osteocalcin within the Tissue}

Using semi-quantitative western blot analysis of the bone marker osteocalcin at week 12 , the bone density was found to be 1.8 times and 2.6 times higher in group III compared to group I and group II, respectively (Figs. 9 and 10).

\section{Statistical Analysis}

A significant difference in bone regeneration was only observed at week 12 for group I (64.5\%), group II $(77.4 \%)$, and group III $(84.8 \%)(p=0.027)$. A post-hoc test to compare the groups was conducted using the
Mann-Whitney test, which uses the Bonferroni correction $(\alpha=0.0167)$. The significance level between group I and group II, group I and group III, and group II and group III was found to be 0.05 at week 12. Although a $P$ value less than 0.05 is considered significant, the significance level did reach the Bonferroni correction $\alpha$ value (0.0167).

\section{Discussion}

The first alveolar bone model using an animal, attempted by Harvold in the 1950s, involved the induction of bone resorption by creating a $2 \mathrm{~mm}$ defect at the alveolar and hard palate of rhesus monkeys. Since then, numerous studies using cat, $\mathrm{dog}$, and rabbit models have been conducted. ${ }^{20-25}$ However, previous studies using medium- and large-sized animal models involved limited sample sizes due to high cost. Also, studies on critical-size bone defects have not yet been conducted. Warren et al. studied bone regeneration by creating $7 \times 4 \times 1.5$ $\mathrm{mm}$ alveolar bone defects in Sprague-Dawley rats, and clarifying the critical size of alveolar bone defects in this model. ${ }^{26}$ These authors found that mature osteoids appear in the artificially-created alveolar defect at week 8 and pass through an inflammation stage and a period of bone remodeling. Formation of bone cells from such osteoids was observed at week 12 through tissue analysis of the bone defect. However, that study was conducted to observe the results of gingivoperiosteoplasty as a treatment for alveolar cleft and a bone-substituting substance was therefore not used to fill in the bone defect. 


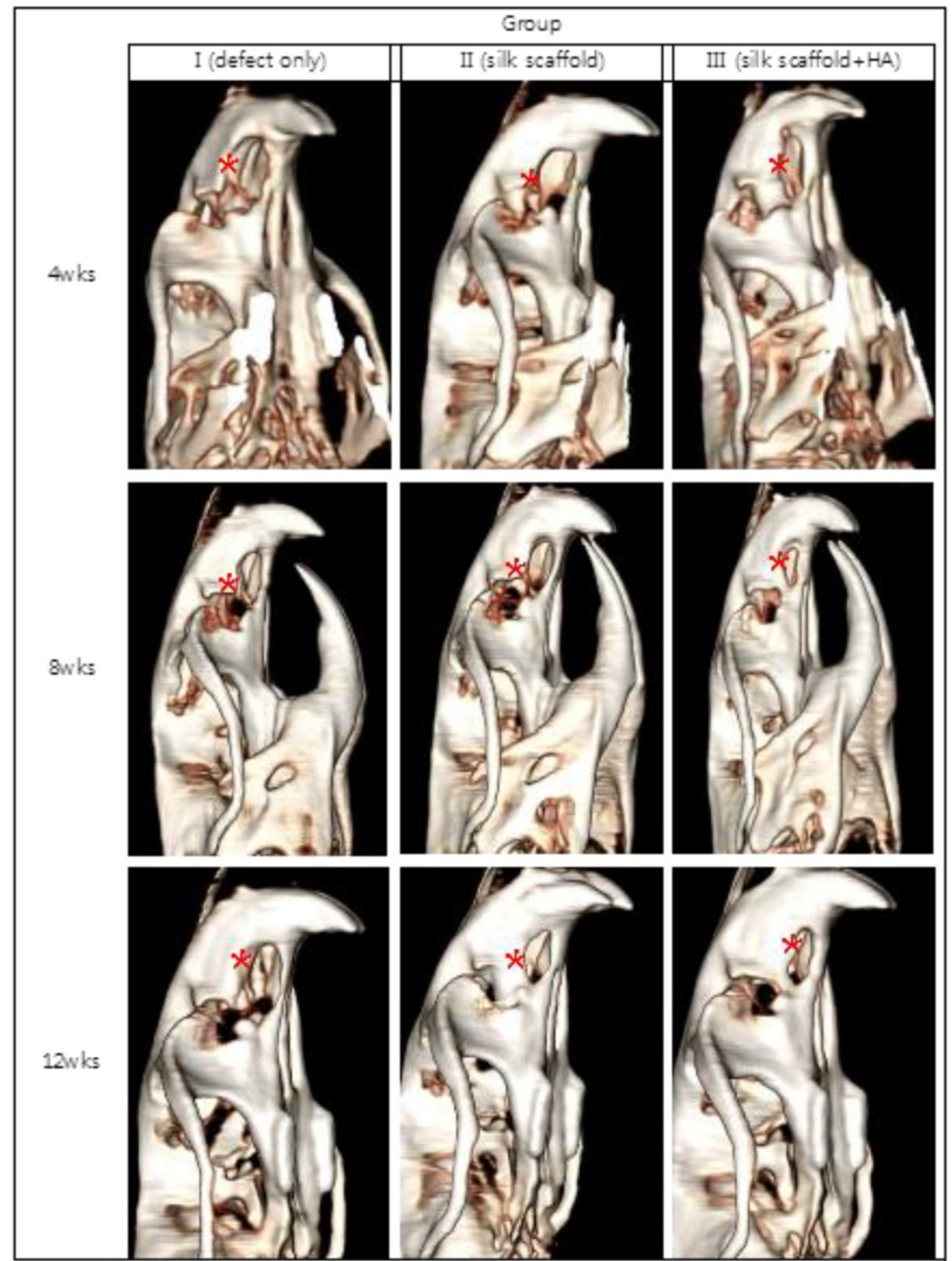

Figure 6. Image of the cranial bone defect reconstructed three-dimensionally after CT. Images were taken at weeks 4,8 , and 12 for each of the specimens in group I, group II, and group III. More bone generation was observed in group II and group III compared to group I, in which little bone generation in the bone defect area was achieved, even at week 12 (*:bone defect area).

Many earlier studies on bone-substituting substances used to fill in a bone defect have been conducted on a variety of bone defect sizes that occurred due to fractures or acute and chronic bone-related damage. Substances for bone defect treatments, both in existence and still under development, can generally be divided into autogenous bone, allogeneic bone, and synthetic substances. However, in order to overcome the shortcomings of previous methods, studies on new approaches that combine bioengineered substances with biological substances, such as growth factors or stem cells, have been conducted. Among these new methodologies, new bone generation using a scaffold with multi-porosity is one of the important technologies that is being used as a substitute in bone defects. ${ }^{27}$ As scaffolding is related to new bone generation, natural materials using synthetic high molecular substances that are biodegradable, such as polyglycolic acid, polylactic acid, poly(D,L-lactic-co-glycolic acid), and collagen have been used. A fibrin and silk scaffold is one such type of natural fiber. ${ }^{1-4,28-31}$ A scaffold used for tissue generation should possess several characteristics, including proper chemical composition, a multi-porous structure for convenient movement when attached to osteoblasts, and a consistent pore distribution for consistent bone generation after 
biodegradation. A silk scaffold meets these requirements. Silk, created from Bombyx mori, has long been used as a suture material as it has outstanding biocompatibility, biological safety, and biodegradability compared with other materials. ${ }^{7-13} \mathrm{~A}$ silk scaffold can be manufactured using various manufacturing processes, and the size and number of multi-porous holes can be adjusted with the control of salt particles when using the salt extraction method. ${ }^{32-34}$ One of distinctive traits of a silk scaffold is its slow degradation compared to other materials. According to the classification of pharmacopoeia published in the United States, silk is classified as a substance incapable of biodegradation due to the fact that it maintains $50 \%$ of its mechanical traits two months after grafting. Therefore, it ultimately enhances the results of bone regeneration by maintaining the holes used for the growth of cells and necessary tissue longer than other scaffolding materials. ${ }^{35}$ Also, silk is more malleable than other types of scaffolding material, and thus better aesthetic and functional results can be acquired, even in cases of a curved bone defect or a defect with a complex shape. For example, the multi-porous silk sponge used in our present study can easily bend when it is dipped into normal saline solution for several minutes. ${ }^{36-38}$ Another distinctive characteristic of a silk scaffold is that it can be sterilized by various methods. Its shape is not changed at $120^{\circ} \mathrm{C}$ or by the gamma ray irradiation such as that used in our current study. Sterilization using ethylene oxide or ethanol is also applicable. ${ }^{39,40}$ This is the most important advantage of silk over other materials such as collagen when conducting operations on actual human bodies.

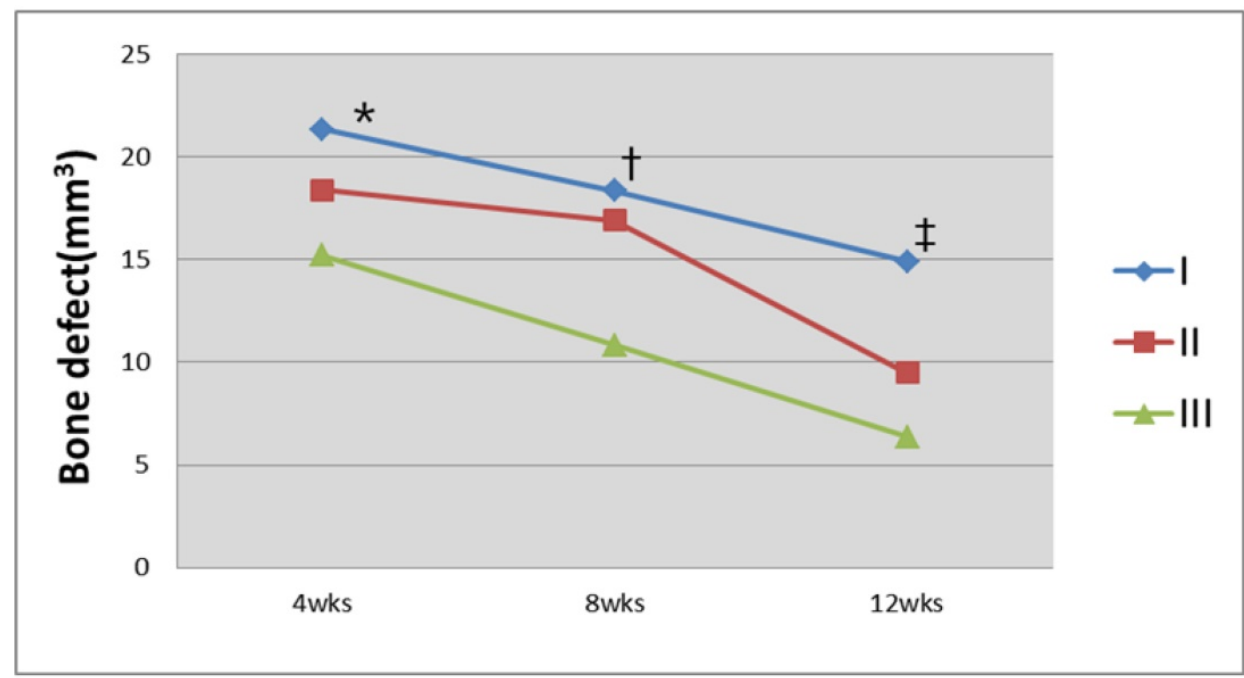

Figure 7. Analysis of the bone defect volume using CT images. Group III had the greatest decrease in the bone defect area 12 weeks after the graft $(* \mathrm{P}=0.058$; $\dagger \mathrm{P}=0.061 ; \ddagger \mathrm{P}=0.027$, Kruskal-Wallis test).

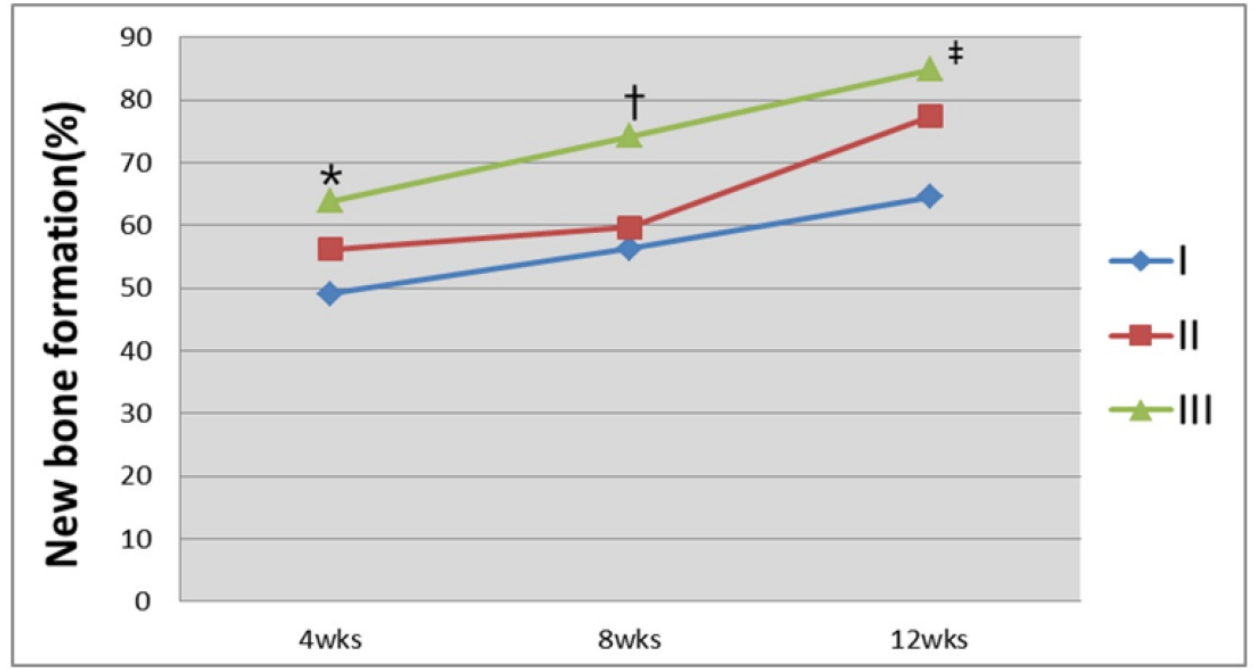

Figure 8. Analysis of the bone regeneration fraction using CT images. Group III was found to have the greatest percentage of bone regeneration 12 weeks after the graft of the hybrid silk and hydroxyapatite scaffold $(* \mathrm{P}=0.058 ; \uparrow \mathrm{P}=0.061 ; \neq \mathrm{P}=0.027$, Kruskal-Wallis test). 


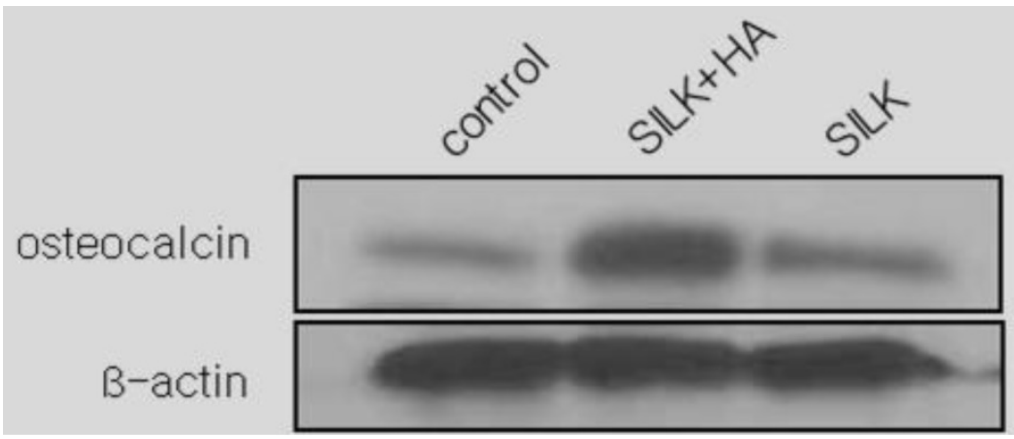

Figure 9. Western blot analysis of osteocalcin 12 weeks after the scaffold graft. The expression level in group III(silk+HA) was higher than in group I (control)or group II(silk).

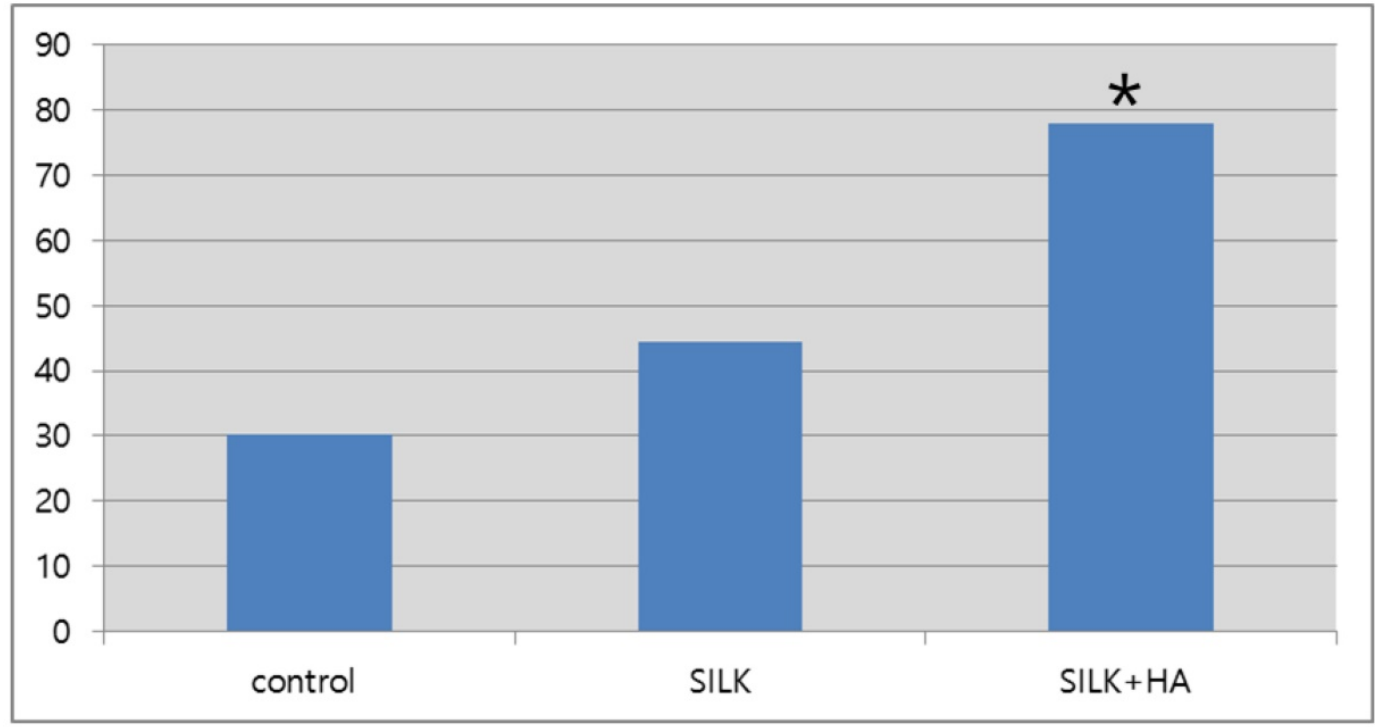

Figure 10. Semi-quantitative expression levels of osteocalcin as determined by western blot 12 weeks after the scaffold graft (control: 30.3; silk: $44.3 ;$ silk+HA; 77.9) $(*: p<0.05)$

Bone regeneration using a hybrid silk and hydroxyapatite scaffold occurs via two processes, osteoconduction from the surrounding bone of the defect area and nucleation with the combined hydroxyapatite acting as a seed. The resulting bone generation from the hybrid scaffold material is faster than the growth generated by the surrounding bone, and the use of the hybrid scaffold brings about consistent ossification in all the affected areas, including the center of the bone defect. ${ }^{15}$

In our current study, bone regeneration was observed only in the area surrounding the bone defect in most of the groups at week 4 and not at the center of the bone defect. More progress in bone regeneration occurred through osteoconduction from the boundaries of the observed bone defects at week 8 . In group III, the bone tissue created from the center of bone defect was observed in a CT cross-section. An increased amount of bone tissue was observed in both the area surrounding the bone defect and the center of the defect at week 12 . This result is significant because the hybrid scaffold induces bone generation using two processes, osteoconduction and osteoinduction.

As a result of calculating the volume of the bone defect and the fraction of the new bone regeneration, the bone defect could reconstruct three-dimensionally using CT. These images showed significant differences in bone regeneration between our study groups at week 12 . Bone regeneration values were $64.5 \%, 77.4 \%$, and $84.8 \%$ in group I, group II, and group III, respectively $(p=0.027)$. Thus, we concluded that more bone regeneration occurred in group III than in the other two groups.

The post-hoc test was conducted using the Mann-Whitney test, which uses the Bonferroni correction ( $\alpha=0.0167)$ to compare our three study groups. The significance level between group I and group II, between group I and group III, and between group II and group III was less than 0.05 at week 12 . A $\mathrm{P}$ value of 0.05 is considered significant, but in this study the significance level of the $\alpha$ value $(0.0167)$ was not reached. This can occur when the number of animals in each group is small compared to the total number of animals used in the study. Therefore, 
additional studies with larger sample sizes are needed to more clearly examine and determine the differences between such groups.

From the cross-section image analysis of CT scans at week 12, both bone regeneration from the boundary of the bone defect and radiolucency in the area surrounding the center of the bone were clearly observed in group III. Measurement of the Hounsfield number (a quantity commonly used in computed tomography (CT) scanning to express CT numbers in a standardised and convenient form) gave a maximum value of 359 , which is relatively low compared to the value of the surrounding bone, which measured from 600 to 800 . This is due to the fact that the bone density of new bone created by bone regeneration is relatively low compared to the mature bone in the surrounding area. This also quantitatively illustrates that this section is not a bone fragment generated from the operation but is newly generated bone.

A semi-quantitative western blot analysis at week 12 found that osteocalcin expression levels were 1.8 and 2.6 times higher in group III compared with group I and group II, respectively $(p<0.05)$, confirming osteoinduction by the hybrid scaffold at the molecular and cellular levels. There were no complications of infection or other side effects in any of the cases, reflecting the outstanding biocompatibility of silk, which has long been used as an effective biomaterial.

\section{Conclusion}

Using an alveolar bone model in Sprague-Dawley rats, we determined that the degree of bone regeneration with a hybrid scaffold of hydroxyapatite and silk is significantly higher than with silk alone or without a scaffold. In the hybrid silk and hydroxyapatite scaffold, new bone is generated via two processes, osteoinduction and osteoconduction, from the boundary and the center of bone defect. The animal specimens survived without grafting-related complications such as infection, hematoma, ectopic bone regeneration, or other side effects.

\section{Competing Interests}

The authors have declared that no competing interest exists.

\section{References}

1. Bajaj AK, Wongworawat AA, Punjabi A. Management of alveolar clefts. J Craniofac Surg. 2003;14:840-46.

2. Matic DB, Power SM. Evaluating the success of gingivoperiosteoplasty versus secondary bone grafting in patients with unilateral clefts. Plast Reconstr Surg. 2008;121:1343-353.

3. Sato Y, Grayson BH, Garfinkle JS, Barillas I, Maki K, Cutting CB. Success rate of gingivoperiosteoplasty with and without secondary bone grafts compared with secondary alveolar bone grafts alone. Plast Reconstr Surg. 2008;121:1356-369.

4. Levenberg S, Langer R. Advances in tissue engineering. Current Topics in Developmental Biology. (61) 113-134, 2004

5. Ikeuchi M, Dohi Y, Horiuchi K, Ohgushi H, Noshi T, Yoshikawa T, Yamamoto K, Sugimura M. Recombinant human bone morphogenetic protein-2 promotes osteogensisw withing ateolpeptide type I collagen solution by combination with rat cultured marrow cells. J biomed Mater Res (60) 61-60, 2002.

6. Saito N, Okada T et al. Biodegradable poly-D, L-Lactic acidpolyethlene glycol block copolymers as a BMP delivery system for injucing bone. J Bone Joint Surg (83) 92-98, 2001.

7. Sofia S, McCarthy MB, Gronowicz G, Kaplan DL. Functionalized silk-based biomaterials for bone formation. J Biomed Mater Res 2001;54:139-448.

8. AltmanGH, Diaz F, Jakuba C, CalabroT, Horan RL, Chen J, et al. Silk-based biomaterials. Biomaterials 2003;24:401-416.

9. Lee KY, Kong SJ, Park WH, Ha WS, Kwon IC. Effect of surface properties on the antithrombogenicity of silk fibroin/S-carboxymethyl kerateine blend films. J Biomater Sci Polym Ed 1998;9:905-14.

10. Santin M, Motta A, Freddi G, Cannas M. In vitro evaluation of the inflammatory potential of the silk fibroin. J Biomed Mater Res 1999;46:382-69.

11. Panilaitis B, Altman GH, Chen J, Jin HJ, Karageorgiou V, Kaplan DL. Macrophage responses to silk. Biomaterials 2003;24:3079-85.

12. Lam KH, Nijenhuis AJ, Bartels H, Postema AR, Jonkman MF, Pennings AJ. Reinforced poly (L-lactic acid) fibres as suturematerial. J Appl Biomater 1995;6:191-7.

13. Rossitch Jr E, Bullard DE, Oakes WJ. Delayed foreign-body reaction to silk sutures in pediatric neurosurgical patients. Childs Nerv Syst 1987;3:375-8.

14. Hutmacher DW, Schantz JT, Lam CXF, et al. State of the art and future directions of scaffold-based bone engineering from a biomaterials perspective. J Tissue Eng Regen Med. 2007;1(4):245-260.

15. Bhumiratana S, Grayson WL, Castaneda A, Rockwood DN, Gil ES, Kaplan DL, Vunjak Novakovic G. Nucleation and growth of mineralized bone matrix on silk-hydroxyapatite composite scaffolds. Biomaterials. 2011 Apr;32(11):2812-20.

16. Na K, Kim SW, Sun BK, Woo DG, Yang HN, Chung HM, et al. Osteogenic differentiation of rabbit mesenchymal stem cells in thermo-reversible hydrogel constructs containing hydroxyapatite and bone morphogenic protein-2 (BMP-2). Biomaterials 2007;28(16):2631e7.

17. Bucholz RW, Carlton A, Holmes RE. Hydroxyapatite and tricalcium phosphate bone graft substitutes. Orthop Clin North Am 1987;18(2):323e34.

18. Radin SR, Ducheyne P. Effect of bioactive ceramic composition and structure on in vitro behavior. III. Porous versus dense ceramics. J Biomed Mater Res 1994;28(11):1303e9.

19. Redey SA, Razzouk S, Rey C, Bernache-Assollant D, Leroy G, Nardin M. Osteoclast adhesion and activity on synthetic hydroxyapatite, carbonated hydroxyapatite, and natural calcium carbonate: relationship to surface energies. J Biomed Mater Res 1999;45(2):140e7.

20. Chierici G, Harvold EP, Dawson WJ. Morphologic adaptations secondary to the production of experimental cleft palate in primates. Cleft Palate J. 1970;7:59-7.

21. El-Deeb M, Horswell B, Waite DE. A primate model for producing experimental alveolar cleft defects. J Oral Maxillofac Surg. 1985;43:523-27.

22. El-Bokle D, Smith SJ, Germane N, Sharawy M. New technique for creating permanent experimental alveolar clefts in a rabbit model. Cleft Palate Craniofac J. 1993;30:542-47.

23. Freng A. Single layered periosteoplasty in experimental midpalatal clefts: A histological study in the cat. Scand J Plast Reconstr Surg. 1979;13:401-08.

24. Griffioen FM, Smit-Vis JH, Urbanus NA. Facial growth in the rabbit after autologous grafting in unilateral clefts. Cleft Palate J. 1988;25:226-34.

25. Jonsson G, Stenstrom S. Maxillary growth after palatal surgery: An experimental study on dogs. Scand J Plast Reconstr Surg. 1978;12:131-37.

26. Nguyen $\mathrm{PD}$, Lin $\mathrm{CD}$, Allori AC, Ricci JL, Saadeh PB, Warren SM. Establishment of a critical-sized alveolar defect in the rat: A model for human gingivoperiosteoplasty. Plast Reconstr Surg. 2009;123:817-25.

27. Ma PX, Zhang R, Xiao G, Franceschi R. Engineering new bone tissue in vitro on highly porous poly(a-hydroxy acids)/hydroxyapatite composite scaffolds. I Biomed Mater Res 2001;54:284-493.

28. Sikavitsas VI, Bancroft GN, Mikos AG. Formation of three-dimensional cell/polymer constructs for bone tissue engineering in a spinner flask and a rotating wall vessel bioreactor. I Biomed Mater Res 2002;62:136-248.

29. Ochi K, Chen G, Ushida T, Gojo S, Segawa K, Tai H, et al. Use of isolated mature osteoblasts in abundance acts as desired-shaped bone regeneration in combination with a modified poly-DL-lactic-aco-glycolic acid (PLGA)-collagen sponge. J Cell Physiol 2003;194:45-953.

30. Yamada Y, Boo JS, Ozawa R, Nagasaka T, Okazaki Y, Hata K, et al. Bone regeneration following injection of mesenchymal stem cells and fibrin glue with a biodegradable scaffold. I Cranio-maxillofac Surg 2003;31:27-133.

31. Rocha LB, Goissis G, Rossi MA. Biocompatibility of anionic collagen matrix as scaffold for bone healing. Biomaterials 2002;23:449-356.

32. Maquet V, Blacher S, Pirard R, Pirard JP, Jérôme R. Characterization of porous polylactide foams by image analysis and impedance spectroscopy. Langmuir 2000;16: 10463-470.

33. Kim UJ, Park J, Kim HJ, Wada M, Kaplan DL. Three-dimensional aqueous-derived biomaterial scaffolds from silk fibroin. Biomaterials 2005;26:2775-85. 
34. Nazarov R, Jin HJ, Kaplan DL. Porous 3-D scaffolds from regenerated silk fibroin. Biomacromolecules 2004;5:718-26.

35. Horan RL, Antle K, Collette AL, Wang Y, Huang J, Moreau JE, et al. In vitro degradation of silk fibroin. Biomaterials 2005;26(17):3385-3.

36. Meinel L, Karageorgiou V, Hofmann S, Fajardo R, Snyder B, Li C, et al. Engineering bone-like tissue in vitro using human bone marrow stem cells and silk scaffolds. J Biomed Mater Res 2004;71A:25-134.

37. Meinel L, Hofmann S, Karageorgiou V, Zichner L, Langer R, Kaplan DL. Engineering cartilage-like tissue using human mesenchymal stem cells and silk protein scaffolds. Biotechnol Bioeng 2004;88:379-891.

38. Wang Y, Kim U-J, Blasioli DJ, Kim HJ, Kaplan DL. In vitro cartilage tissue engineering with $3 \mathrm{D}$ porous aqueous-derived silk scaffolds and mesenchymal stem cells. Biomaterials 2005;26:7082-.94.

39. Karageorgiou V, Meinel L, Hofmann S, Malhotra A, Volloch V, Kaplan D. Bone morphogenetic protein-2 decorated silk fibroin films induce osteogenic differentiation of human bone marrow stromal cells. J Biomed Mater Res A 2004;71(3):528-7.

40. Li C, Vepari C, Jin HJ, Kim HJ, Kaplan DL. Electrospun silk-BMP-2 scaffolds for bone tissue engineering. Biomaterials 2006;27(16):3115-4. 